\title{
Prevalence of Tick Infestation in Bovine in Gozamine Woreda NorthWeast, of Ethiopia
}

\author{
Tadele Laikun Alemineh Shime \\ Amhara regional state East Gojjam zone Gozamin woreda livestock and fishery development office
}

\begin{abstract}
A cross sectional study was conducted from April 2016 to June 2016 in Gozamin district to determine the prevalence of tick infestation in bovine. Ticks were identified by their features by stereomicroscope. in current study adult ticks were collected from 384 local and cross breed cattle and examined of which $274971.35 \% 0$ animal were infested by with different genera of tics. The major tick genera identified by this study were Amblypomma, Hyaloma and Boophilus with prevalence of 189(49.2\%), 103(26.6\%), 78(20.3\%), and 58(15.1\%) respectively. The prevalence of Amblypomma and Boophilus in young bovine were 61(43.9\%) and 23(16.5\%) respectively. the prevalence of Amblyomma and Boophilus in adult cattle were 128(52.2\%) and $35(14.3 \%)$ respectively. There was statically significance $(\mathrm{p}<0.05)$ in age. Similarly the prevalence of Amblyomma in male and female was $134(57.8 \%)$ and 55(36.2\%) respectively but prevalence of Boophilus was40 (17.2\%) and $18(11.8 \%)$ respectively there Was statically difference on sex $(\mathrm{p}<0.05)$.the prevalence of Amblyomma in extensive and semi intensive production system was $173(57.9 \%$ ) and $1618.8 \%$ ) whereas the prevalence of Boophilus was $49(16.4 \%)$ and $9(10.6 \%)$. The prevalence of the same genera in poor medium and good body condition were78 $(61.7 \%)$ $39(29.3 \%)$ and $24(19 \%)$. The result of the present study reveal that there was high tick infestation in the study area. Therefore appropriate control measure should de implemented.
\end{abstract}

Keyword: bovine, Gozamin, prevalence, tick

DOI: $10.7176 / \mathrm{JNSR} / 9-11-03$

Publication date:June $30^{\text {th }} 2019$

\section{Introduction}

Ethiopia has approximately 47.57 million cattle and 52.3 million sheep and goat. Cattle play significant role in the socioeconomic life of the people of Ethiopia. In addition to the product of meat and milk cattle provide drought power for cultivation of crop. Skin and hide were also important components of livestock sector in generating foreign export earnings (CSA, 2008).

In Ethiopia tick occupy the first place among external parasites and economic loss tick born disease are the major constraint in the genetic improvement of by cross breeding of local cattle with exotic breed (Fesha,1983).

Ixodid ticks are one of the most important external parasites of livestock on the tropical and sub-tropical pare of the world because of direct and indirect effect on their host ticks are considered to be not only a significant threat to successful livestock production, but also serious interfere with economy of the country especial in Africa (Decastro, 1997).tics occur are worldwide there was about 852 species of tick in the world that parasitizing both animal and human (Walker etal., 2003). In Ethiopia 47 species of tick were identified and have effect on skin and hide (Kassa, 2005).

Tick can be directly cause illness, toxicities and tick paralysis (Brossard, 1998). The indirect pathogenic effect of tick were transmission of disease from infected animal to health animal such as babesiosis, east coast fever and cowdrosis (FAO, 1984).

The successful implementation of susceptible tick control program in grazing animal is depend on knowledge of the epidemiology of the parasites due to interaction with the host in the specific climate management and production. In some countries substantial ecological and epidemiological knowledge base has been established through extensive studies and field trials on contrary developing countries including Ethiopia lack such information due to insufficient human economic and infrastructure (FAO, 2004).

The objectives of this study was

$>$ To determine the prevalence of tick and associated risk factor in Gozamine worwda.

\section{Material and methods \\ Study area}

The study was conduct in North West of Ethiopia in Gozamin woreda in east gojam zone. It has 3 agro ecological zone high land, weynadega and lowland and the woreda has 111234 sheep and goat 123456 cattle, 31678 equine and 89789 poultry (GWAO, 2015).

\section{Study population}

Cattle that found in the woreda were consider as study population. 


\section{Study design}

A cross-sectional study was implemented to determine the prevalence of tick genera in bovine from April 2016 to June 2016 in Gozamine woreda.

Sample size and sampling method

The sample size was determined by using thurs field (2001).

$\mathrm{N}=\underline{1.96^{2} \mathrm{p}(1-\mathrm{p})}$

$$
\mathrm{D}^{2}
$$

Where $\mathrm{n}=$ sample size required

$\mathrm{Z}=$ taken from the level for $95 \% \operatorname{ci}(1.96)$

$\mathrm{D}=$ the level of precision $(5 \%)$

$\mathrm{P}=$ expected prevalence (50) since there is no previous study.

Based on this the sample size was 384 and the sampling method was random sampling.

\section{Tick collection}

Ticks were collected from predilection site like neck ear perineum udder inguinal area tips of tail by using forceps. The sample were preserved and labeled and examine at Gozamine woreda veterinary clinic by stereomicroscope.

\section{Data analysis}

All date recorded in this study were coded and entered in Microsoft spread sheet. Date analyzed by using verson 19 satirical soft were.

\section{Results}

During the study total of 384 cattle were examined of which $274(71.35 \%)$ animals were infested with different genera of tick.

Tabell the prevalence of tick based on age

\begin{tabular}{lllll}
\hline Age & Total animal examined & Tick genera & & \\
\hline & & Amblyomma & Boophilu & Hyaloma \\
Young & 139 & $61(43.9 \%)$ & $23(16.5 \%)$ & $18(12.9 \%)$ \\
adult & 245 & $128(52.2 \%)$ & $35(14.3 \%)$ & $60(24.5)$ \\
total & 384 & $189(49.2 \%)$ & $58(15.1 \%)$ & $78(20.3 \%)$ \\
P value & 0.005 & & & \\
\hline
\end{tabular}

Similarly the prevalence of this different genus of tick between the different sexes of cattle was also recorded the highest prevalence was observed male than female for Amblyomma, Hyalomma and Boophilus respectively. The prevalence of sex in male and female was $32.4 \%$ and 21.2 respectively. The result showed significant difference observed $(\mathrm{p}<0.05)$

Table 2 the prevalence of tick in and around Gozamine woreda based on sex

\begin{tabular}{lllll}
\hline sex & Total no of animal examined & Tick genera & & \\
\hline & & Amblyomma & $\begin{array}{l}\text { Hyalo } \\
\text { mma }\end{array}$ & Boophilus \\
male & 232 & $134(57.8 \%)$ & $61(26.3 \%)$ & $40(17.2 \%)$ \\
female & 152 & $55(36.2 \%)$ & $17(11.2 \%)$ & $18(11.8 \%)$ \\
total & 384 & $189(49.2 \%)$ & $78(20.3 \%)$ & $58(15.1 \%)$ \\
P value & 0.001 & & & \\
\hline
\end{tabular}

Similarly the prevalence of age and sex, the prevalence of different genus of tick between breed of cattle were also record. The highest prevalence was observed in local than cross for Amblyomma, Hyalomma, and Boophilus. the result showed that significant different observed $(\mathrm{p}<0.05)$.

Table 3 the prevalence of cattle tick in Gozamine based on breed

\begin{tabular}{lllll}
\hline breed & Total no animal examind & Tick genera & & \\
\hline & & Amblyomma & Hyalomma & Boophilus \\
local & 251 & $139(55.4 \%)$ & $45(17.9 \%)$ & $33(13 \%)$ \\
cross & 133 & $50(37.6 \%)$ & $33(24.8 \%)$ & $25(18.8 \%)$ \\
total & 384 & $189(49.2 \%)$ & $78(20.3 \%)$ & $58(15.1 \%)$ \\
P value & 0.03 & & & \\
\hline
\end{tabular}

In addition the prevalence of different genus of tick between management system of cattle were also record the highest prevalence was observed in extensive than intensive for Amblyomma, Hyalomma and Boophilus respectively. The prevalence of management in extensive and intensive $31.5 \%$ and $15.0 \%$ respectively. The result showed that significance difference observed $(\mathrm{p}<0.05)$. 
Table 4 the prevalence of tick cattle in Gozamine based on management

\begin{tabular}{lllll}
\hline Management system & Total no animals examined & Tick genera & & \\
\hline & & Amblyomma & Hyalomma & Boophilus \\
extensive & 299 & $173(57.9 \%)$ & $66(22.1 \%)$ & $49(16.4 \%)$ \\
semi intensive & 85 & $16(18.8 \%)$ & $12(14.21 \%)$ & $9(10.6 \%)$ \\
total & 384 & $189(49.2 \%)$ & $78(20.3 \%)$ & $58(15.1 \%)$ \\
P value & 0.001 & & & \\
\hline
\end{tabular}

Based on the body condition score of animal, the prevalence of the different genera of tick was identified with the highest prevalence of tick in poor, medium and good body condition respectively. The prevalence of body condition score in poor, medium and good was $13.9 \%, 31.8 \%$ and $18.4 \%$ respectively. The result showed that significance difference observed $(\mathrm{p}<0.05)$.

Table 5 the prevalence of cattle tick in Gozamine based in body condition

\begin{tabular}{lllll}
\hline Body condition score & Total no of animal examind & Tick genra & & \\
\hline & & Amblyomma & Hyalomma & Boophilus \\
good & 133 & $39(29.3 \%)$ & $16(12 \%)$ & $15(11.3 \%)$ \\
medium & 125 & $72(57.6 \%)$ & $29(23.2 \%)$ & $19(15.2 \%)$ \\
poor & 126 & $78(61.9 \%)$ & $33(26.2 \%)$ & $24(19 \%)$ \\
total & 384 & $189(49.2 \%)$ & $78(20.3 \%)$ & $58(15.1 \%)$ \\
P value & 0.002 & & & \\
\hline
\end{tabular}

\section{Discussion}

The current study revealed that the overall prevalence of tick was 274(71.35\%) in bovine examined in the study period. The tick was identified at genera level and was Amblyomma, Hyalomma and Boophilus, 189(49.2\%), $103(26.6 \%)$ and $78(20.3 \%)$ respectively. The higher prevalence was recorded in Amblyomma which is comparable with Seyoum (2005) who reported 55.9\%, 5.3 and 3.85 for Amblyomma, Hyalomma and Boophilus respectively in eastern Amara and second was Hyalomma which is comparable with Endale (2006) with prevalence of 31.4\% in ambo region. This might be due to seasonal variation geographical location and time of collection. The prevalence of other genera was 20.3 for boophilus which is not comparable with Sebsebie (1998), Hilina (2010), Seyoum (2005) and Endale (2006) with rate of 2.6\%,5.4\%, 5.3 and .14\% in sedamo in and around mekele, eastern amhara and in abo area of Ethiopia respectively. This might be due geographical location.

The least genera was identified during the study period was boophiluis which account for rates of $15.5 \%$ which is not comparable with study of Ataklty (2007) and Endale (2006) with rate of 53.9\% and 54.5\% respectively.

Prevalence tick was $63.5 \%$ and $31.5 \%$ in extensive and semi intensive production system respectively which is agreed with the work of Heilina (2010) was reported 58.6\% and 29.1 in in extensive and semi intensive production system. This due to the animal has not got chance to grazing area for cross breed where as extensive management animals have got chance to tick in grazing land.

Tick infestation has a great variation in sex in which females are more infected than male this due to females' immune system lowered during lactation and pregnancy. Higher prevalence was recorded in adult animal than young animal this due to young animal keep in the house which do not got the tick.

\section{Conclusion and recommendation}

Gozamin woreda has large number of livestock which contribute for cash income, drought power, and milk and meat production. The present study revealed that different tick genera has been identified such Amblyomma, Hyalomma, and Boophilus which has a great effect on the production system of the woreda cattle which cause loss of body weight, decreased milk and meat production and hide and skin production. Based on the above conclusion the following recommendations are recommended.

$>$ The woreda must be implemented appropriate control programme such regular spraying of cattle by using accaricides.

$>$ Create awareness for the farmer for the control program of tick

\section{Reference}

Ataakilty, h.2007.stud on the prevakence of bovine ectoparasite of cattle in and around mekele.dvm thesis, fm aau debreziet ,ethipa.

Barnett, s.f.19671. The control of tick on livestock fao.agric, .sec.,pp.54.

Baailu, a.2004. A study of tick and tick born protozoan in cattle at assela, aresi zone dvm thesis fvm,aau debereziet ethiopa.

Bram,r.a. 1983. Tick born livestock diseases and vector. The global problem in tick and tick born Disease, fao. 
Animal production and health

Brossard, m.1998 the use of vaccine and genetically resistant animals in tick control.rev.sci.tech.off.int.epiz 17(1)pp188-199.

CSA, 2008. Federaldemocratic republic of ehiopia agricuklture sample survey,volume 11 report On livestovk addis abrba.pp. 183 .

Cumming ,g.s1991.host distribution do notlimit the species range of most Africa tickl bulletin of entomologicalreserarch.pp.303-327.

Decastro,j.j. 1991. Rtesistant to ixodid in cattle with an assement of it role in tick control in Africa. Pp.244-262.

GWAO. 2015. Gozamin woreda agriculture office.

FAO, 1984.tick and tick born disease control .a practical manual .vol.1, pp.229

FAO, 2004.tick and tick born disease control .a practical manual .vol.1, pp.176.

Fasha, g.1983.note on tick species and tickl born diseases of domestic animals in Ethiopia.FVM, AAU,debrezeite,Ethiopia.

Hilena,b.2010. a survey of tick on cattle in and around mekele. Dvm theiss. FVM, AAU,debrezeite,Ethiopia.

Jongestan,f. and uilnberg,g.19994. ectoparasires of animal control method. Rev.sec.tech.off.int.epiz.,13(4),pp.1201-1226.

Kassa, b.2005.standard veterinary laboratory diagnosic manual volume 3.moa, Addis Ababa.

Katherin,m.k 1979 tick of veterinary importance . animal and plant health inspection service, united department of agriculture. Pp1-122.morel,p 10989. Manual of tropical veterinary parasitology. Tick born diseases of livestock in Africa. Cabinternational,uk. Pp 299-460.

Seyum, z.2005 distribution and host parasitic relationship of ixodid tick in eastern amhara, Ethiopia, kombolcha regional veterinary laboratory,kombolicha. EthiopiA, VET. JOURNAL,9(1).9-17.

Tthistfiel, 2001. Veterinary epidemiology.3rded.blackwellpp233.

Walker, j.b.1974.the ixodid ticks if Kenya. Areview of presnt knowledge of their host and Distribution common wealth instiyute of entomolgy, London.pp220.

Yitebark,g.2004 tick species infesting livestock in jimma area ,sout weast of etghiopia. Dvm theiss. FVM, AAU,debrezeite,Ethiopia. 